\title{
田 \\ ON SOME RIESZ AND CARLEMAN TYPE INEQUALITIES FOR HARMONIC FUNCTIONS ON THE UNIT DISK
}

\author{
DAVID KALAJ AND ELVER BAJRAMI
}

\begin{abstract}
We prove some isoperimetric type inequalities for real harmonic functions in the unit disk belonging to the Hardy space $h^{p}, p>1$ and for complex harmonic functions in $h^{4}$. The results extend some recent results on the area. Further we discus some Riesz type results for holomorphic functions.
\end{abstract}

\section{INTRODUCTION AND STATEMENT OF MAIN RESULTS}

Throughout the paper we let $\mathbf{U}=\{z \in \mathbb{C}:|z|<1\}$ be the open unit disk in the complex plane $\mathbb{C}$, and let $\mathbf{T}=\{z \in \mathbb{C}:|z|=1\}$ be the unit circle in $\mathbb{C}$. The normalized area measure on $\mathbf{U}$ will be denoted by $d \sigma$. In terms of real (rectangular and polar) coordinates, we have

$$
d \sigma=\frac{1}{\pi} d x d y=\frac{1}{\pi} r d r d \theta, \quad z=x+i y=r e^{i \theta} .
$$

For $0<p<+\infty$ let $L^{p}(\mathbf{U}, \sigma)=L^{p}$ denote the familiar Lebesgue space on $\mathbf{U}$ with respect to the measure $\sigma$. The Bergman space $A^{p}(\mathbf{U})=A^{p}$ is the space of all holomorphic functions in $L^{p}(\mathbf{U}, \sigma)$. For a fixed $1 \leq p<+\infty$, denote by $A_{0}^{p}$ the set of all functions $f \in A^{p}$ for which $f(0)=0$. For a function $f$ in $L^{p}(\mathbf{U}, \sigma)$, we write

$$
\|f\|_{p}=\left(\int_{\mathbf{U}}|f(z)|^{p} d \sigma\right)^{1 / p}
$$

Bergman space $b^{p}$ of harmonic functions is defined similarly.

The Hardy space $h^{p}$ is defined as the space of (complex) harmonic functions $f$ such that

$$
\|f\|_{h^{p}}:=\sup _{0 \leq r<1}\left(\int_{0}^{2 \pi}\left|f\left(r e^{i t}\right)\right|^{p} \frac{d t}{2 \pi}\right)^{1 / p}<\infty .
$$

If $f \in h^{p}$, then by [1, Theorem 6.13], there exists

$$
f\left(e^{i t}\right)=\lim _{r \rightarrow 1} f\left(r e^{i t}\right) \text {, a.e. }
$$

and $f \in L^{p}(\mathbf{T})$. It can be shown that there hold

$$
\|f\|_{h^{p}}^{p}=\lim _{r \rightarrow 1} \int_{0}^{2 \pi}\left|f\left(r e^{i t}\right)\right|^{p} \frac{d t}{2 \pi}=\int_{0}^{2 \pi}\left|f\left(e^{i t}\right)\right|^{p} \frac{d t}{2 \pi} .
$$

Similarly we define the Hardy space $H^{p}$ of holomorphic functions.

File: kalbaj.tex, printed: 2021-7-27, 23.28

${ }^{1} 2010$ Mathematics Subject Classification: Primary 47B35

Key words and phrases. Harmonic functions, Ries inequality, Isoperimetric inequality. 
The starting point of this paper is the well known isoperimetric inequality for Jordan domains and isoperimetric inequality for minimal surfaces due to Carleman [3]. In that paper Carleman, among the other results proved that if $u$ is harmonic and smooth in $\overline{\mathbf{U}}$ then

$$
\int_{\mathbf{U}} e^{2 u} d x d y \leq \frac{1}{4 \pi}\left(\int_{0}^{2 \pi} e^{u} d t\right)^{2}
$$

By using a similar approach as Carleman, Strebel ([13]) proved the isoperimetric inequality for holomorphic functions; that is if $f \in H^{1}(\mathbf{U})$ then

$$
\int_{\mathbf{U}}|f(z)|^{2} d x d y \leq \frac{1}{4 \pi}\left(\int_{\mathbf{T}}\left|f\left(e^{i t}\right)\right| d t\right)^{2} .
$$

This inequality has been proved independently by Mateljević and Pavlović ([11]). In [6] have been done some generalizations for the space.

In this paper we prove the following results.

Theorem 1.1. Let $f$ be a real harmonic function. For $p>1$ we have that

$$
\|f\|_{b^{2 p}} \leq C_{p}\|f\|_{h^{p}}
$$

where

$$
C_{p}=M_{2 p}= \begin{cases}\frac{\cos \frac{\pi}{4 p}}{\cos \frac{\pi}{2 p}}, & \text { if } 1<p \leq 2 ; \\ \frac{\cos \frac{p}{4 p}}{\sin \frac{\pi}{2 p}}, & \text { if } p \geq 2 .\end{cases}
$$

The inequality (1.2) extends the main result in [2], where Bajrami proved the same result but only for $p=4$. But the wrong constant appear in [2], due to a wrong citation to the Duren approach [5, p. 67-68]. However the same method produces the same constant namely $C_{4}=\frac{1}{2 \sin \frac{\pi}{16}} \approx 2.56292$. We were not able to check if (1.2) is sharp. We are thankful to professor Hedenmalm who drawn attention to his paper [7], where is treated a problem which suggests that the inequalities from Theorem 2.1, which we use in order to prove (1.2), are maybe not sharp. The inequality (1.2) improves similar results for real harmonic functions proved by Kalaj and Meštrović in [10] and by Chen and Ponnusamy and Wang in [4]. We expect that the inequality (1.2) is true for complex harmonic mappings for every $p>1$. Kalaj and Meštrović in [10] proved it for $p=2$, namely they obtained that $C_{2}=\frac{1}{2 \sin \frac{\pi}{8}} \approx 1.3$. On the same paper the example $f_{a}(z)=\Re \frac{z}{1-a z}$, when $a \uparrow 1$ produces the constant $C_{0}=(5 / 2)^{1 / 4} \approx 1.257$, so the constant $C_{2}$ is not far from the sharp constant. In this paper we extend it for $p=4$.

Namely we have

Theorem 1.2. If $f \in h^{4}(\mathbf{U})$ is a nonzero complex harmonic function then $f \in b^{8}$ and there hold the inequality

$$
\|f\|_{b^{8}} \leq \frac{1}{2 \sin \frac{\pi}{16}}\|f\|_{h^{4}}
$$

The main ingredient of the proofs are some sharp M. Riesz type inequalities proved for Hardy space by Verbitsky ([14]): 
Proposition 1.3 (Riesz type inequality). Let $p>1$. For every holomorphic mapping there hold the sharp inequalities

$$
L_{p}\|\Re f\|_{h^{p}} \leq\|f\|_{h^{p}} \leq R_{p}\|\Re f\|_{h^{p}}
$$

provided $\left|\arg f(0)-\frac{\pi}{2}\right| \geq \frac{\pi}{2 \bar{p}}$, or $f(0)=0$, where $\bar{p}=\max \left\{p, \frac{p}{p-1}\right\}$ and

$$
R_{p}=\left\{\begin{array}{ll}
\frac{1}{\cos \frac{\pi}{2 p}}, & \text { if } 1<p \leq 2 ; \\
\frac{1}{\sin \frac{\pi}{2 p}}, & \text { if } p \geq 2 .
\end{array} \quad \text { and } L_{p}= \begin{cases}\frac{1}{\sin \frac{\pi}{2 p}}, & \text { if } 1<p \leq 2 ; \\
\frac{1}{\cos \frac{\pi}{2 p}}, & \text { if } p \geq 2 .\end{cases}\right.
$$

In addition we refer to the references [8] and [12] for related results.

By results of Verbitsky we prove some similar inequalities for Bergman space (Theorem 2.1).

From Proposition 1.3, we obtain that, if $f=u+i v$ is holomorphic with $f(0)=$ 0 , then

$$
\|f\|_{H^{p}}^{p} \leq \frac{R_{p}^{p}}{2}\left(\|u\|_{h^{p}}^{p}+\|v\|_{h^{p}}^{p}\right)
$$

and

$$
\frac{L_{p}^{p}}{2}\left(\|u\|_{h^{p}}^{p}+\|v\|_{h^{p}}^{p}\right) \leq\|f\|_{H^{p}}^{p} .
$$

Now we formulate a similar result, where $\frac{R_{p}^{p}}{2}$ and $\frac{L_{p}^{p}}{2}$ are replaced by smaller, respectively bigger constants for $p$ close to 4 .

Theorem 1.4. Let $p \geq 2$ and let $f=u+i v$ be a holomorphic function on the unit disk so that $f(0)=0$ and let $f \in H^{p}$. Then for $p \in[2,4]$ we have

$$
\|f\|_{H^{p}} \leq\left(\frac{p}{p-1}\right)^{1 / p}\left(\|u\|_{h^{p}}^{p}+\|v\|_{h^{p}}^{p}\right)^{1 / p}
$$

and for $p \geq 4$ we have

$$
\|f\|_{H^{p}} \geq\left(\frac{p}{p-1}\right)^{1 / p}\left(\|u\|_{h^{p}}^{p}+\|v\|_{h^{p}}^{p}\right)^{1 / p} .
$$

Remark 1.5. If $C_{p}=\left(\frac{p}{p-1}\right)^{1 / p}$, then $2^{-1 / p} L_{p} \leq C_{p}$ if $p \geq 4$ and $C_{p} \leq 2^{-1 / p} R_{p}$, if $p_{1} \leq p \leq 4$, so inequalities (1.5) and (1.6) improve the inequalities (1.3) and (1.4), if $p_{1} \leq p \leq 4$, and if $p \geq 4$, respectively. Here $p_{1} \approx 2.42484$ is the only solution of the equation

$$
\left(\frac{p}{p-1}\right)^{1 / p}=2^{-1 / p} \frac{1}{\sin \frac{\pi}{2 p}}, \quad p \geq 2 .
$$

The proofs are presented in sections 2 and 3 and 4 . 


\section{Proof of Theorem 1.1}

From Proposition 1.3 we obtain

$$
L_{p}^{p} \int_{\mathbf{T}}\left|\Re f\left(r e^{i t}\right)\right|^{p} d t \leq \int_{\mathbf{T}}\left|f\left(r e^{i t}\right)\right|^{p} d t \leq R_{p}^{p} \int_{\mathbf{T}}\left|\Re f\left(r e^{i t}\right)\right|^{p} d t .
$$

So

$$
\begin{aligned}
L_{p}^{p} \int_{0}^{1} r d r \int_{\mathbf{T}}\left|\Re f\left(r e^{i t}\right)\right|^{p} d t & \leq \int_{0}^{1} \int_{\mathbf{T}} r\left|f\left(r e^{i t}\right)\right|^{p} d t \\
& \leq R_{p}^{p} \int_{0}^{1} r d r \int_{\mathbf{T}}\left|\Re f\left(r e^{i t}\right)\right|^{p} d t .
\end{aligned}
$$

Thus we have the following theorem

Theorem 2.1. Let $p>2$ and $f=\Re f+i \Im f \in b^{p}(\mathbf{U})$. If $\left|\arg f(0)-\frac{\pi}{2}\right| \geq \frac{\pi}{2 p}$, or $f(0)=0$, then we have

$$
L_{p}\|\Re f\|_{b^{p}} \leq\|f\|_{b^{p}} \leq R_{p}\|\Re f\|_{b^{p}}
$$

and

$$
L_{p}\|\Im f\|_{b^{p}} \leq\|f\|_{b^{p}} \leq R_{p}\|\Im f\|_{b^{p}} .
$$

From now on we will use the shorthand notation

$$
\int_{\mathbf{T}} f:=\frac{1}{2 \pi} \int_{0}^{2 \pi} f\left(e^{i t}\right) d t
$$

and

$$
\int_{\mathbf{U}} f:=\frac{1}{\pi} \int_{\mathbf{U}} f(z) d x d y, \quad z=x+i y .
$$

Thus for $p>2$ we have

$$
\begin{aligned}
\left(\int_{\mathbf{U}}|\Re f|^{p}\right)^{1 / p} & \leq \frac{1}{L_{p}}\left(\int_{\mathbf{U}}|f|^{p}\right)^{1 / p} \\
& \leq \frac{1}{L_{p}}\left(\int_{\mathbf{T}}|f|^{p / 2}\right)^{2 / p} \\
& \leq \frac{R_{p / 2}}{L_{p}}\left(\int_{\mathbf{T}}|\Re f|^{p / 2}\right)^{2 / p} \\
& =M_{p}\left(\int_{\mathbf{T}}|\Re f|^{p / 2}\right)^{2 / p}
\end{aligned}
$$

where

$$
M_{p}= \begin{cases}\frac{\cos \frac{\pi}{2 p}}{\cos \frac{\pi}{p}}, & \text { if } 2<p \leq 4 \\ \frac{\cos \frac{\pi}{2 p}}{\sin \frac{n}{p}}, & \text { if } p \geq 4 .\end{cases}
$$

This finishes the proof of Theorem 1.1 


\section{Proof of Theorem 1.2}

We assume that $f(z)=g(z)+\overline{h(z)}$, where $h(0)=0$, and $g$ and $h$ are holomorphic on the unit disk.

Lemma 3.1. The function $|a|^{2}+|b|^{2}$ is log-subharmonic, provided that $a$ and $b$ are analytic.

Proof. We need to show that $f(z)=\log \left(|a|^{2}+|b|^{2}\right)$ is subharmonic. By calculation we find

and so

$$
f_{z}=\frac{a^{\prime} \bar{a}+b^{\prime} \bar{b}}{|a|^{2}+|b|^{2}}
$$

$$
\begin{aligned}
& f_{z \bar{z}}=\frac{a^{\prime} \bar{a}^{\prime}+b^{\prime} \bar{b}^{\prime}}{|a|^{2}+|b|^{2}}-\frac{a^{\prime} \bar{a}+b^{\prime} \bar{b}}{|a|^{2}+|b|^{2}} \frac{a \bar{a}^{\prime}+b \bar{b}^{\prime}}{|a|^{2}+|b|^{2}} \\
& =\frac{\left(\left|a^{\prime}\right|^{2}+\left|b^{\prime}\right|^{2}\right)\left(|a|^{2}+|b|^{2}\right)-\left|\bar{a} a^{\prime}+\bar{b} b^{\prime}\right|^{2}}{\left(|a|^{2}+|b|^{2}\right)^{2}}
\end{aligned}
$$

which is clearly positive.

Now from the isoperimetric inequality for log-subharmonic functions (e.g. [9] Lemma 2.2]), we have

Lemma 3.2. For every positive number $p$ and analytic functions $a$ and $b$ defined on the unit disk $U$ we have that

$$
\int_{\mathbf{U}}\left(|a|^{2}+|b|^{2}\right)^{2 p} \leq\left(\int_{\mathbf{T}}\left(|a|^{2}+|b|^{2}\right)^{p}\right)^{2} .
$$

Further let

$$
L=\int_{\mathbf{U}}\left(|g+\bar{h}|^{2}\right)^{4}=\int_{\mathbf{U}}\left(|g|^{2}+|h|^{2}+2 \Re(g h)\right)^{4} .
$$

Then

$$
\begin{aligned}
L & =\sum_{k=0}^{4}\left(\begin{array}{l}
4 \\
k
\end{array}\right) \int_{\mathbf{U}}\left(|g|^{2}+|h|^{2}\right)^{k}(2 \Re(g h))^{4-k} \\
& \leq \sum_{k=0}^{4}\left(\begin{array}{l}
4 \\
k
\end{array}\right) \int_{\mathbf{U}}\left(\left(|g|^{2}+|h|^{2}\right)^{4}\right)^{k / 4}\left(\int_{\mathbf{U}}|2 \Re(g h)|^{4}\right)^{(4-k) / 4} .
\end{aligned}
$$

Let $E_{4}=\cos \frac{\pi}{8}$. From Lemma 3.2 and Theorem 2.1 we have

$$
\begin{aligned}
L & \leq \sum_{k=0}^{4}\left(\begin{array}{l}
4 \\
k
\end{array}\right) E_{4}^{4-k}\left(\int_{\mathbf{U}}\left(|g|^{2}+|h|^{2}\right)^{4}\right)^{k / 4}\left(\int_{\mathbf{U}}(2|g h|)^{4}\right)^{(4-k) / 4} \\
& \leq \sum_{k=0}^{4}\left(\begin{array}{l}
4 \\
k
\end{array}\right) E_{4}^{4-k}\left(\int_{\mathbf{T}}\left(|g|^{2}+|h|^{2}\right)^{4 / 2}\right)^{2 k / 4}\left(\int_{\mathbf{T}}(2|g h|)^{4 / 2}\right)^{2(4-k) / 4} .
\end{aligned}
$$


Further we have

$$
\begin{aligned}
X: & =\int_{\mathbf{T}}|g+\bar{h}|^{4} \\
& =\int_{\mathbf{T}}\left(|g|^{2}+|h|^{2}+2 \Re(g h)\right)^{2} \\
& =\int_{\mathbf{T}}\left(|g|^{2}+|h|^{2}\right)^{2}+4\left(|g|^{2}+|h|^{2}\right) \Re(g h)^{2}+4(\Re(g h))^{2} \\
& =\int_{\mathbf{T}}\left(|g|^{2}+|h|^{2}\right)^{2}+4\left(|g|^{2}+|h|^{2}\right) \Re(g h)+2|g h|^{2} .
\end{aligned}
$$

Let

and

$$
A=\left(\int_{\mathbf{T}}\left(|g|^{2}+|h|^{2}\right)^{2}\right)^{1 / 2}
$$

Then

$$
B=\left(\int_{\mathbf{T}} 4|g|^{2}|h|^{2}\right)^{1 / 2} .
$$

$$
\begin{aligned}
\left|\int_{\mathbf{T}}\left(|g|^{2}+|h|^{2}\right) \Re(g h)\right| & \leq \mid\left(\int_{\mathbf{T}}\left(\left(|g|^{2}+|h|^{2}\right)\right)^{2}\right)^{1 / 2}\left(\int_{\mathbf{T}}|g h|^{2} / 2\right)^{1 / 2} \\
& =A B \cdot \frac{\sqrt{2}}{4} .
\end{aligned}
$$

Further we have

$$
X \geq A^{2}+\frac{B^{2}}{2}-\sqrt{2} A B=\left(A-\frac{\sqrt{2}}{2} B\right)^{2} .
$$

Furthermore

$$
A^{2}-B^{2}=\int_{\mathbf{T}}\left(|g|^{2}-|h|^{2}\right)^{2} \geq 0
$$

and thus

$$
X \geq\left(\frac{2-\sqrt{2}}{2}\right)^{2} B^{2}
$$

and similarly

$$
X \geq\left(\frac{2-\sqrt{2}}{2}\right)^{2} A^{2} .
$$

Hence

$$
\begin{aligned}
\int_{\mathbf{U}}(|g+\bar{h}|)^{8} & \leq \sum_{k=0}^{4}\left(\begin{array}{l}
4 \\
k
\end{array}\right) E_{4}^{4-k}\left(\frac{2}{2-\sqrt{2}}\right)^{4}\left(\int_{\mathbf{T}}|g+\bar{h}|^{4}\right)^{2} \\
& =\left(\frac{2\left(1+E_{4}\right)}{2-\sqrt{2}}\right)^{4}\left(\int_{\mathbf{T}}|g+\bar{h}|^{4}\right)^{2} .
\end{aligned}
$$


So

$$
\|g+\bar{h}\|_{b^{8}} \leq \sqrt{\frac{2\left(1+E_{4}\right)}{2-\sqrt{2}}}\|g+\bar{h}\|_{h^{4}},
$$

where $E_{4}=\cos \left[\frac{\pi}{8}\right]=\frac{\sqrt{2+\sqrt{2}}}{2}$. Finally we have that

$$
\|g+\bar{h}\|_{b^{8}} \leq \frac{1}{2 \sin \frac{\pi}{16}}\|g+\bar{h}\|_{h^{4}}
$$

Here $\frac{1}{2 \sin \frac{\pi}{16}} \approx 2.56292$. This finishes the proof of Theorem 1.2

\section{Proof of Theorem 1.4}

We use the following form of Green formula

$$
r \int_{0}^{2 \pi} \frac{\partial G\left(r e^{i t}\right)}{\partial r} d t=\int_{|z| \leq r} \Delta G(z) d x d y .
$$

Let $p>2$ and let $f=u+i v$ be an analytic function. Let $q=\frac{p}{p-1}$ and $\epsilon>0$ and define

$$
\begin{gathered}
F_{\epsilon}(z)=\left.\left.|q \epsilon+| f(z)\right|^{2}\right|^{p / 2} \\
U_{\epsilon}(z)=\left|\epsilon+u(z)^{2}\right|^{p / 2} \\
V_{\epsilon}(z)=\left|\epsilon+v(z)^{2}\right|^{p / 2}
\end{gathered}
$$

Then by direct calculation we obtain

$$
\begin{aligned}
& \Delta F_{\epsilon}=p\left(q \epsilon+|f|^{2}\right)^{p / 2-2}\left(2 q \epsilon+p|f|^{2}\right)\left|f^{\prime}\right|^{2}, \\
& \Delta U_{\epsilon}=p\left(u^{2}+\epsilon\right)^{p / 2-2}\left|f^{\prime}\right|^{2}\left(\epsilon+(p-1) u^{2}\right),
\end{aligned}
$$

and

$$
\Delta V_{\epsilon}=p\left(v^{2}+\epsilon\right)^{p / 2-2}\left|f^{\prime}\right|^{2}\left(\epsilon+(p-1) v^{2}\right)
$$

If $p=4$, then

$$
\Delta U_{\epsilon}+\Delta V_{\epsilon}=\frac{3}{4} \Delta F_{\epsilon} .
$$

Lemma 4.1. Let $f$ be a holomorphic function defined on the unit disk $\mathbf{U}$. For $p>4$ and $z \in \mathbf{U}$ and $\epsilon>0$ we have

$$
\Delta\left(U_{\epsilon}+V_{\epsilon}\right) \leq\left(\frac{p-1}{p}\right) \Delta F_{\epsilon} .
$$

For $1 \leq p \leq 4$ and $z \in \mathbf{U}$ and $\epsilon>0$ we have

$$
\Delta\left(U_{\epsilon}+V_{\epsilon}\right) \geq\left(\frac{p-1}{p}\right) \Delta F_{\epsilon} .
$$


Proof. Let $f=u+i v=r e^{i s}$ and define

$$
Q(s)=\frac{\Delta\left(U_{\epsilon}+V_{\epsilon}\right)}{\Delta F_{\epsilon}} .
$$

Then from (4.2), (4.3) and (4.4) we have

$$
\begin{gathered}
Q(s)=\frac{\left(\epsilon+r^{2} \cos ^{2} s\right)^{-2+\frac{p}{2}}\left(\epsilon+(p-1) r^{2} \cos ^{2} s\right)}{\left(2 \epsilon+(p-1) r^{2}\right)\left(\frac{\epsilon p}{-1+p}+r^{2}\right)^{-2+\frac{p}{2}}} \\
+\frac{\left(\epsilon+r^{2} \sin ^{2} s\right)^{-2+\frac{p}{2}}\left(\epsilon+(p-1) r^{2} \sin ^{2} s\right)}{\left(2 \epsilon+(p-1) r^{2}\right)\left(\frac{\epsilon p}{-1+p}+r^{2}\right)^{-2+\frac{p}{2}}}
\end{gathered}
$$

We should prove that

First of all

$$
Q(s)\left\{\begin{array}{l}
\geq \text { if } p<4 \\
\leq \text { if } p \geq 4
\end{array}\right.
$$

where

$$
Q^{\prime}(s)=\frac{(-2+p) r^{2}\left(\frac{\epsilon p}{p-1}+r^{2}\right)^{2-\frac{p}{2}} \cos s \sin s}{2 \epsilon+(p-1) r^{2}} T
$$

$$
\begin{aligned}
T & =\left(\epsilon+r^{2} \cos ^{2} s\right)^{-3+\frac{p}{2}}\left(-3 \epsilon+(1-p) r^{2} \cos ^{2} s\right) \\
& +\left(\epsilon+r^{2} \sin ^{2} s\right)^{-3+\frac{p}{2}}\left(3 \epsilon+(p-1) r^{2} \sin ^{2} s\right) .
\end{aligned}
$$

Then $T=0$, if and only if

$$
L=S^{\frac{1}{2}(6-p)}=R:=\frac{3 \epsilon+(p-1) r^{2} \sin ^{2} s}{3 \epsilon+(p-1) r^{2} \cos ^{2} s},
$$

where

$$
S=\frac{\epsilon+r^{2} \sin ^{2} s}{\epsilon+r^{2} \cos ^{2} s} \text {. }
$$

If $p=6$, then $\cos ^{2} s=\sin ^{2} s$. If $p>6$ then we also have $\cos ^{2} s=\sin ^{2} s$, because if for example $\cos ^{2} s>\sin ^{2} s$, then $L>1>R$. Similarly $\cos ^{2} s<\sin ^{2} s$ implies $L<1<R$. If $4<p<6$ and $\cos ^{2} s>\sin ^{2} s$, then

$$
R-S=\frac{\epsilon(4-p) r^{2} \cos (2 s)}{\left(\epsilon+r^{2} \cos ^{2} s\right)\left(3 \epsilon+(p-1) r^{2} \cos ^{2} s\right)}<0 .
$$

Thus

$$
S<R<1 .
$$

Since $0<\frac{6-p}{2}<1$, it follows that

$$
S<R^{\frac{6-p}{2}}<1
$$

So $L \neq R$. If $4<p<6$ and $\cos ^{2} s<\sin ^{2} s$, then 


$$
S>R>1
$$

So

$$
S>R^{\frac{6-p}{2}}
$$

Thus $L \neq R$.

If $p<4$, then $\cos ^{2} s>\sin ^{2} s$ implies that

$$
1>S>R>R^{\frac{6-p}{2}} \text {. }
$$

Finally if $p<4$ and $\cos ^{2} s>\sin ^{2} s$. Then

$$
1<S<R<R^{\frac{6-p}{2}} \text {. }
$$

We proved that the only stationary points of $Q$ are

$$
s_{j}=\frac{\pi j}{4}, \quad j=0, \ldots, 7 .
$$

So wee need to show that $Q\left(s_{j}\right) \leq 1$ for $p \leq 4$ and $Q\left(s_{j}\right) \geq 1$ for $p \geq 4$.

Let $s=0$. Show that

$$
Q(0)=\frac{\left(\epsilon^{-1+\frac{p}{2}} p+p\left(\epsilon+r^{2}\right)^{-2+\frac{p}{2}}\left(\epsilon+(p-1) r^{2}\right)\right)}{p\left(2 \epsilon+(p-1) r^{2}\right)\left(\frac{\epsilon p}{p-1}+r^{2}\right)^{\frac{p}{2}-2}} \begin{cases}\geq 1, & \text { if } p \leq 4 \\ \leq 1, & \text { if } p \geq 4 .\end{cases}
$$

Let $\epsilon=t r^{2}, t>0$. Then

$$
Q(0)=\frac{\left(\left(1+\frac{p t}{p-1}\right)\right)^{2-\frac{p}{2}}\left(t^{-1+\frac{p}{2}}+(1+t)^{-2+\frac{p}{2}}(p-1+t)\right)}{p-1+2 t} .
$$

By using convexity of the function $k(x)=x^{p / 2-2}$, for $p<4$ we have

$$
\frac{t^{-1+\frac{p}{2}}}{p-1+2 t}+\frac{(1+t)^{-2+\frac{p}{2}}(p-1+t)}{p-1+2 t}>\left(\frac{p-1+p t+2 t^{2}}{p-1+2 t}\right)^{-2+\frac{p}{2}} \text {. }
$$

Further

So $Q(0) \geq 1$.

$$
\left(\frac{p-1+p t+2 t^{2}}{p-1+2 t}\right)^{-2+\frac{p}{2}}>\left(\frac{p-1+p t}{p-1}\right)^{-2+\frac{p}{2}} .
$$

For $4 \leq p$ we need to show that

i.e.

$$
\frac{t^{-1+\frac{p}{2}}}{p-1+2 t}+\frac{(1+t)^{-2+\frac{p}{2}}(p-1+t)}{p-1+2 t}<\left(\frac{p-1+p t}{p-1}\right)^{-2+\frac{p}{2}}
$$

$$
\frac{t^{-1+\frac{p}{2}}}{p-1+2 t}+\frac{(1+t)^{-2+\frac{p}{2}}(p-1+t)}{p-1+2 t}<\left(1+t+\frac{t}{p-1}\right)^{-2+\frac{p}{2}} .
$$

The last inequality is equivalent with the trivial inequalities

$$
\frac{t\left(t^{-2+\frac{p}{2}}-(t+1)^{-2+\frac{p}{2}}\right)}{p-1+2 t}<0<\left(1+t+\frac{t}{p-1}\right)^{-2+\frac{p}{2}}-(1+t)^{-2+\frac{p}{2}} .
$$


For $s=\pi / 4$, and $\epsilon=r^{2} t$,

$$
Q(s)=2^{2-\frac{p}{2}}\left(\frac{p-1+p t}{(p-1)(1+2 t)}\right)^{2-\frac{p}{2}} .
$$

So

$$
Q(s) \begin{cases}\geq 1, & \text { if } p \leq 4 \\ \leq 1, & \text { if } p \geq 4\end{cases}
$$

The other cases can be treated in a similar way.

Proof of Theorem 1.4 Assume that $p \leq 4$. By using the Green formula (4.1), and Lemma 4.1 we obtain

$$
\begin{aligned}
r \int_{0}^{2 \pi} \frac{\partial}{\partial r} F_{\epsilon} d t & =\int_{0}^{r} \int_{0}^{2 \pi} \rho \Delta F_{\epsilon} d \rho d t . \\
& \leq \frac{p}{p-1} \int_{0}^{r} \int_{0}^{2 \pi} \rho\left(\Delta U_{\epsilon}+\Delta V_{\epsilon}\right) d \rho d t \\
& =r \frac{p}{p-1} \int_{0}^{2 \pi} \frac{\partial}{\partial r}\left(U_{\epsilon}+V_{\epsilon}\right) d t .
\end{aligned}
$$

Dividing by $r$ and integrating in $[0,1]$ with respect to $r$ we obtain

$$
\begin{aligned}
\int_{0}^{2 \pi}\left[F_{\epsilon}\left(e^{i t}\right)\right. & \left.-F_{\epsilon}(0)\right] d t \\
& \leq \frac{p}{p-1}\left(\int_{0}^{2 \pi}\left[U_{\epsilon}\left(e^{i t}\right)-U_{\epsilon}(0)\right] d t+\int_{0}^{2 \pi}\left[V_{\epsilon}\left(e^{i t}\right)-V_{\epsilon}(0)\right]\right) d t .
\end{aligned}
$$

Letting $\epsilon \rightarrow 0$ we obtain

$$
\int_{0}^{2 \pi}\left|f\left(e^{i t}\right)\right|^{p} d t \leq \frac{p}{p-1}\left(\int_{0}^{2 \pi}\left|u\left(e^{i t}\right)\right|^{p} d t+\int_{0}^{2 \pi}\left|v\left(e^{i t}\right)\right|^{p}\right) d t .
$$

Similarly we prove the related inequality for $p \geq 4$.

\section{REFERENCES}

[1] S. Axler, P. Bourdon, W. Ramey, Harmonic function theory, Springer Verlag New York 1992.

[2] E. BAJRAMI, Improvement of isoperimetric type inequality for harmonic functions in the case $p=4$, to appear in Indagationes Mathematicae, http://dx.doi.org/10.1016/j.indag.2016.10.003.

[3] T. CARleman, Zur Theorie der Minimalfächen. (German) Math. Z. 9 (1921), no. 1-2, 154-160.

[4] Sh. Chen, S. Ponnusamy, And X. Wang, The isoperimetric type and Fejer-Riesz type inequalities for pluriharmonic mappings, Scientia Sinica Mathematica (Chinese Version), 44(2)(2014), 127-138.

[5] P. L. DuREn, Theory of $H^{p}$ spaces. Pure and Applied Mathematics, Vol. 38 Academic Press, New York-London 1970 xii+258 pp.

[6] F. HAng, X. WANG, X. YAn, Sharp integral inequalities for harmonic functions. Comm. Pure Appl. Math. 61 (2008), no. 1, 54-95.

[7] H. Hedenmalm, Bloch functions, asymptotic variance, and geometric zero packing, arXiv: 1602.03358

[8] B. Hollenbeck, I. E. Verbitsky, Best constants for the Riesz projection. J. Funct. Anal. 175 (2000), no. 2, 370-392. 
[9] D. KalaJ, Isoperimetric inequality for the polydisk. Ann. Mat. Pura Appl. (4) 190 (2011), no. 2, 355-369

[10] D. KALAJ, R. MEŠTroviĆ, Isoperimetric type inequalities for harmonic functions. J. Math. Anal. Appl. 373 (2011), no. 2, 439-448.

[11] M. Mateljević, M. Pavlović, New proofs of the isoperimetric inequality and some generalizations. J. Math. Anal. Appl. 98 (1984), no. 1, 25-30.

[12] S. K. PICHORIDES, On the best values of the constants in the theorems of M. Riesz, Zygmund and Kolmogorov. Collection of articles honoring the completion by Antoni Zygmund of 50 years of scientific activity, II. Studia Math. 44 (1972), 165-179.

[13] K. Strebel: Quadratic differentials. Ergebnisse der Mathematik und ihrer Grenzgebiete (3), 5. Springer-Verlag, Berlin, 1984. xii+184 pp.

[14] I. E. VERBITSKY, Estimate of the norm of a function in a Hardy space in terms of the norms of its real and imaginary parts. Linear operators. Mat. Issled. No. 54 (1980), 16-20, 164-165.

Faculty of Natural Sciences and Mathematics, University of Montenegro, Cetinjski put B.B. 81000 Podgorica, Montenegro

E-mail address: davidk@ac.me

Department of Mathematics, University of Prishtina, Mother Teresa, No. 5, 10000, PrishtinA, Kosovo

E-mail address: elver.bajrami@uni-pr.edu 